\title{
Evaluation of meridional scans for angle closure assessment with anterior segment swept-source optical coherence tomography
}

\author{
Natalia Porporato, ${ }^{1}$ Mani Baskaran, ${ }^{1}$ Shamira Perera, ${ }^{1}$ Tin A Tun, ${ }^{1}$ Rehena Sultana, ${ }^{2}$ \\ Marcus Tan, ${ }^{3}$ Joanne HuiMin Quah, ${ }^{4}$ John C Allen, ${ }^{2}$ David Friedman, ${ }^{5}$ Ching Yu Cheng, ${ }^{1}$ \\ Tin Aung
}

${ }^{1}$ Glaucoma, Singapore Eye Research Institute/Singapore National Eye Centre, Singapore ${ }^{2}$ Centre for Quantitative Medical School, Singapore ${ }^{3}$ Ophthalmology, National University Hospital, National University of Singapore, Singapore

${ }^{4}$ Outram Polyclinic, SingHealth Polyclinics, Singapore ${ }^{5}$ Ophthalmology, Harvard University, Cambridge, Massachusetts, USA

Correspondence to Singapore National Eye Centre Singapore 168751, Singapore; aung.tin@snec.com.sg

Received 28 October 2019 Revised 28 January 2020 Accepted 20 February 2020 Published Online First 9 March 2020 Medicine, Duke-NUS Graduate Dr Tin Aung, Glaucoma,

\begin{abstract}
Background/aims As swept-source optical coherence tomography (SS-OCT) simultaneously obtains 128 meridional scans, it is important to identify which scans are playing the main role in classifying gonioscopic angle closure to simplify the analysis. We aimed to evaluate the diagnostic performance of every meridional scan in its ability to detect gonioscopic angle closure.

Methods Observational study with 2027 phakic subjects consecutively recruited from a community polyclinic. Gonioscopy and SS-OCT were performed. Gonioscopic angle closure was defined as non-visibility of the posterior trabecular meshwork in $\geq 180^{\circ}$ of the angle, while SS-OCT was defined as iridotrabecular contact anterior to the scleral spur. The area under the receiver operating characteristic curve (AUC) was calculated to assess the diagnostic performance of each single scan, the sequential anticlockwise cumulative effect of those single scans and different combinations of them.

Results The AUCs of each scan ranged from 0.73 to 0.82 . The single scan at $80^{\circ}-260^{\circ}$ had the highest AUC $(0.82,95 \% \mathrm{Cl} 0.79$ to 0.84$)$ and performed significantly better than most of the temporonasal scans (from $0^{\circ}$ to $52^{\circ}$ and from $153^{\circ}$ to $\left.179^{\circ}\right)$. The superoinferior scans achieved higher AUCs compared with the temporonasal ones. When assessing the cumulative effect of adding individual scans consecutively, the peak AUC (0.80) was obtained when considering the superoinferior scans closer to $80^{\circ}-85^{\circ}$, but no further positive cumulative effect was seen when adding the rest of the temporonasal scans of the circumference.

Conclusions In conclusion, the single SS-OCT scan at $80^{\circ}-260^{\circ}$ had the highest diagnostic performance. Our study suggests that the $360^{\circ}$ evaluation may not translate to better clinical utility for detection of gonioscopic angle closure.
\end{abstract}

Check for updates

(C) Author(s) (or their employer(s)) 2021. No commercial re-use. See rights and permissions. Published by BMJ.

To cite: Porporato $\mathrm{N}$ Baskaran M, Perera S, et al. Br J Ophthalmol

2021:105:131-134.
Anterior segment optical coherence tomography (AS-OCT) technology has become a popular tool for assessment of angle closure in clinical settings. AS-OCT offers several advantages in comparison with the current reference standard, the gonioscopy, for angle assessment. Apart from being a recordable method, its non-contact nature may lead to a better compliance among physicians and patients. AS-OCT findings are also less dependent on observer's training. However, it requires good image quality to be able to identify the landmarks for classification.

Time domain AS-OCT (Visante; Carl Zeiss Meditec, Dublin, California, USA) obtains one to four meridional scans of the anterior chamber angle at a time. Commonly, one vertical (corresponding to $\left.90^{\circ}-270^{\circ}\right)$ and one horizontal $\left(0^{\circ}-180^{\circ}\right)$ scan is assessed. It is limited by up to $30 \%$ ungradable angle images, mainly due to poor localisation of the scleral spur. ${ }^{1}$ Compared with time domain AS-OCT, the swept-source optical coherence tomography (SS-OCT, CASIA SS-1000; Tomey Corporation, Nagoya, Japan) has higher resolution and fewer ungradable images $(8 \%){ }^{2}$ SS-OCT simultaneously captures 128 meridional scans (256 angle images) of the entire angle circumference in $2 \mathrm{~s}$. We have shown in a previous work that SS-OCT has a good diagnostic performance for detecting gonioscopic angle closure using either 16 automatically graded circumferential scans per eye (area under the receiver operating characteristic curve (AUC) 0.83$)^{3}$ or 128 manually graded scans (AUC 0.84$).{ }^{2}$ While $360^{\circ}$ circumferential anterior segment imaging provides a more comprehensive understanding of angle status, SS-OCT is limited by the requirement to manually identify the scleral spur in a number of meridional scans, a process that is time-consuming.

The aim of this study was to evaluate the best single meridional SS-OCT scan to classify gonioscopic angle closure. This could simplify incorporation of SS-OCT into clinical practice.

\section{METHODS}

\section{Study population and recruitment}

Subjects aged $\geq 50$ years with no previous history of glaucoma, intraocular surgery or ocular trauma were consecutively recruited from a Singapore community polyclinic providing primary healthcare services. From June to September 2013, a total of 2038 consecutive subjects were eligible and screened for this study. We previously reported that 92\% (1857) of the included subjects had gradable SS-OCT images; $87.8 \%$ were Chinese with a mean age of $61.8 \pm 6.7$ years; and $63.5 \%$ were women. ${ }^{2}$

\section{SS-OCT imaging}

Before any contact procedure, all subjects underwent SS-OCT imaging under dark room conditions. Gentle manipulation of the upper and lower 


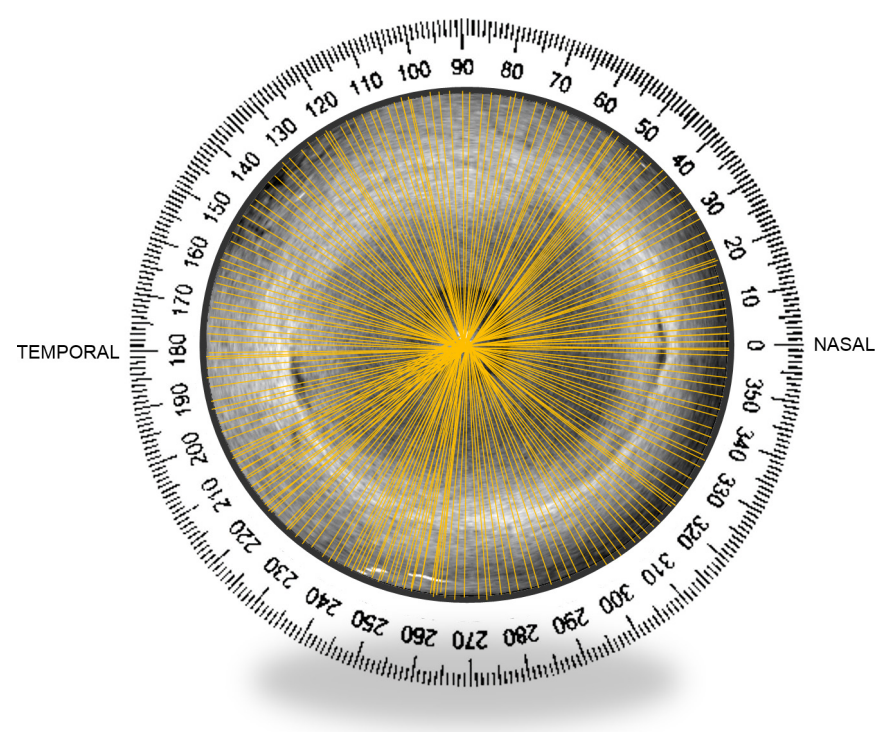

Figure 1 SS-OCT $360^{\circ}$ analysis. Localisation of the SS-OCT meridional scans in the right eye. SS-OCT, swept-source optical coherence tomography.

eyelid was applied by a second person for better visualisation, with special care to avoid inadvertent pressure on the globe. Patients were asked to focus on an internal fixation target, and once the patient had been optimally positioned, each eye was scanned with the three-dimensional angle analysis scan (which takes $2.4 \mathrm{~s}$ ) using the autoalignment function. The algorithm acquires 128 consecutive meridional scans, each consisting of 512 A-scans across the anterior chamber.

All 128 meridional images (256 angles) were assessed by a single trained ophthalmologist (NP) who was masked to gonioscopy findings. We have previously reported good intraobserver and interobserver agreement for image grading using SS-OCT. ${ }^{4}$ A closed angle on an SS-OCT image was defined as contact between the iris and any part of the angle wall anterior to the scleral spur. An SS-OCT meridional scan was considered closed when either of the two angles assessed was closed. We have considered single scans instead of single angles as the software provides meridionals for analysis. Figure 1 shows the localisation of the SS-OCT meridionals scans in the right eye.

\section{Gonioscopy}

Indentation gonioscopy using a Sussman 4-mirror goniolens (Ocular Instruments, Bellevue, Washington, USA) was performed in the dark by a glaucoma fellowship-trained ophthalmologist (MT) who was masked to imaging results. A weighted kappa of 0.82 was achieved in the assessment of angle grading when comparing with the readings of an ophthalmologist with subspecialty glaucoma training (TA). ${ }^{4}$ A $1 \mathrm{~mm}$ light beam was reduced to a narrow slit, and the vertical beam was offset horizontally for evaluating nasal and temporal angles and was maintained vertically for assessing superior and inferior angles. The examination was performed with the subject's eye in the primary position of gaze. Care was taken to avoid light from falling on the pupil and to avoid inadvertent indentation during examination. In some cases, the gonioscopy lens was tilted slightly to allow a view of the angle over the convexity of the iris, avoiding ocular distortion. The angle in each quadrant was considered 'closed' if the posterior pigmented trabecular meshwork could not be seen in the primary position without indentation. An eye was classified as having angle closure if there were two or more closed quadrants. In this large sample, the distributions of gonioscopic angle closure by quadrant were $8.62 \%(160 / 1857), 4.63 \%(86 / 1857)$, $6.35 \%(118 / 1857)$ and $6.83 \%(127 / 1857)$, for the superior, inferior, temporal and nasal quadrants, respectively.

\section{Statistical analysis}

Only right eyes were taken for analysis to avoid intereye correlation. AUCs with 95\% CIs were calculated to assess the performance of every individual SS-OCT scan, the sequential anticlockwise cumulative effect of individual scans and different scan combinations using gonioscopy as the reference standard. A $\chi^{2}$ test was used for comparison of AUCs. Statistical significance was set at $\mathrm{p}<0.05$ and SAS V.9.4 statistical software was used.

\section{RESULTS}

One hundred and thirty-nine right eyes from the 1857 individuals in this study had $\geq 2$-quadrant gonioscopic angle closure (7.5\% prevalence). Figure 2 shows the diagnostic performance of each of the 128 scans $\left(0^{\circ}-180^{\circ}\right.$ to $\left.179^{\circ}-359^{\circ}\right)$ individually. The scan $80^{\circ}-260^{\circ}$ had the highest AUC $(0.82$, 95\% CI 0.79 to 0.84 ) to classify gonioscopic angle closure and performed significantly better than most of the temporonasal scans (from $0^{\circ}$ to $180^{\circ}$ to $52^{\circ}-232^{\circ}$ and from $153^{\circ}$ to $333^{\circ}$ to $179^{\circ}-359^{\circ}$ ). The AUCs of individual scans ranged from 0.73 to 0.82 , with superoinferior scans showing higher AUCs compared with the temporonasal ones. The scan $19^{\circ}-199^{\circ}$ had the lowest AUC $(0.73,95 \%$ CI 0.69 to 0.78 ).

Figure 3 shows the cumulative effect of individual scans on AUC. The peak AUC $(0.80)$ was obtained by summing the superoinferior scans to $80^{\circ}-85^{\circ}$; however, no additional benefit to AUC was accrued from summing temporonasal circumferential scans beyond $85^{\circ}$ as diagnostic performance dropped substantially.

The combination of the single horizontal $\left(0^{\circ}-180^{\circ}\right)$-vertical $\left(90^{\circ}-270^{\circ}\right)$ scans, the combination of the diagonal scans $\left(45^{\circ}-\right.$ $225^{\circ}$ and $135^{\circ}-315^{\circ}$ ) or the combination of the four of them resulted in an AUC of approximately 0.80. However, they performed significantly worse than the single $80^{\circ}-260^{\circ}$ scan $(\mathrm{p}<0.001)$ (table 1).

\section{DISCUSSION}

We found that the single SS-OCT scan between $80^{\circ}$ and $260^{\circ}$ had the highest diagnostic performance (AUC 0.82) to detect gonioscopic angle closure, performing better than the most commonly used combinations of single scans $(p<0.001)$. This result was almost as good as manually grading 128 meridians $^{2}$ when compared with gonioscopy as the reference standard. Our findings suggest that it may be unnecessary to manually evaluate all 128 SS-OCT scans to classify gonioscopic angle closure eyes based on a qualitative assessment. This novel finding may simplify using SS-OCT in clinical practice as not all scans would need to be manually marked. Further research needs to be done in order to validate these findings in other AS-OCT devices. However, AS-OCT technology remains suboptimal to perfectly discriminate angle closure based on gonioscopy, and the results did not differ much whether single or multiple scans were analysed.

We previously reported an AUC of $0.84^{2}$ for the manual grading of the 128 scans (256 angles) that are imaged by SS-OCT and AUC of 0.83 for 16 meridional scans (32 angles) graded by an in-built software (iris-trabecular contact index). ${ }^{3}$ This performance resulted better than the one found in the literature for Visante in an equivalent community-based 

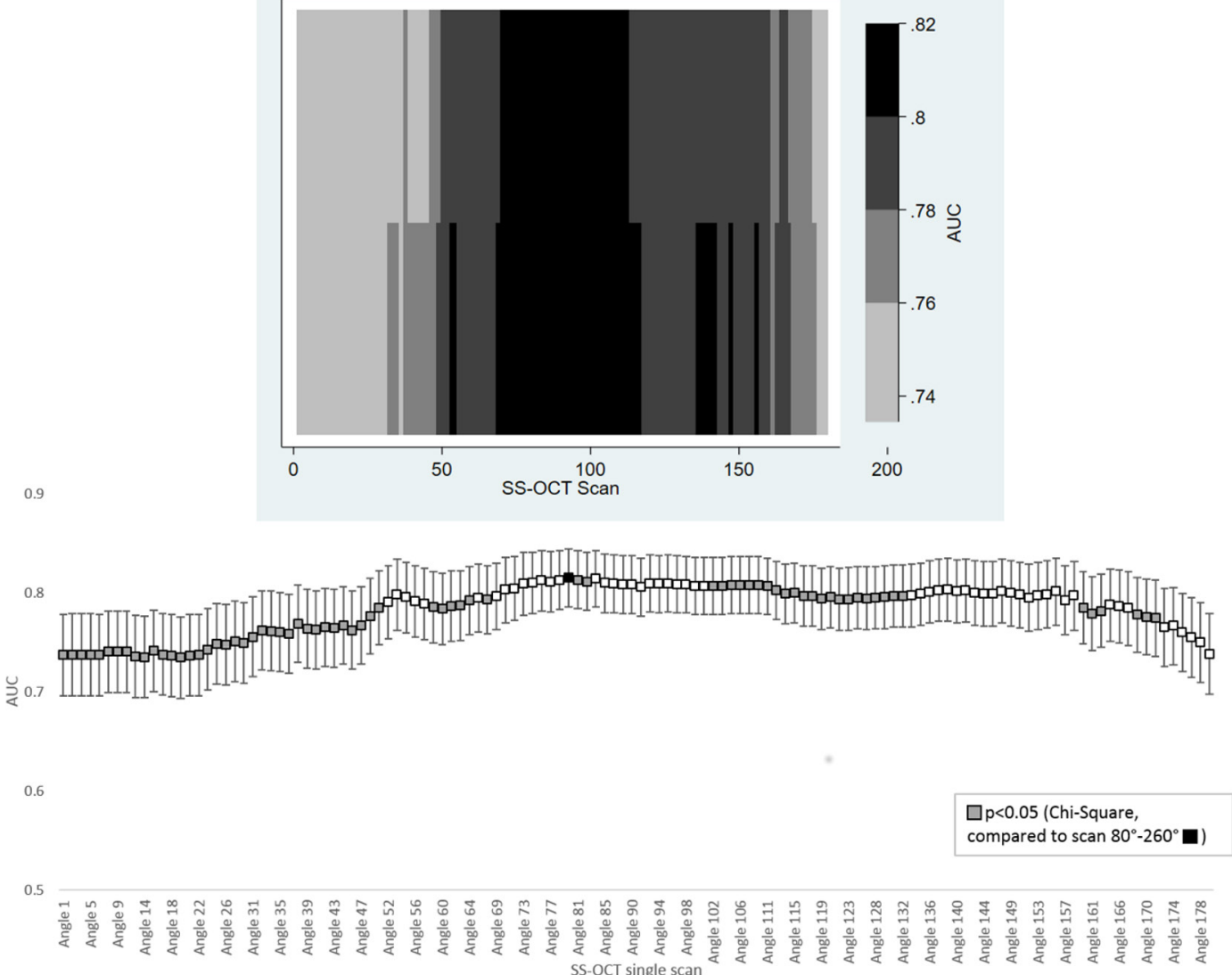

Figure 2 Diagnostic performance of SS-OCT single scans for two-quadrant gonioscopic angle closure. Heat map (top) and dot plot (bottom) showing the AUC for the 128 individual SS-OCT scans representing the $360^{\circ}$ of the circumference. AUC, area under the receiver operating characteristic curve; SS-OCT, swept-source optical coherence tomography.

qualitative study (AUC 0.76). ${ }^{5}$ However, several studies have shown better results with Visante AS-OCT. Tun $e t$ al $^{6}$ reported an AUC of 0.90 in a tertiary eye clinic setting. Another two studies analysed Visante quantitative parameters in nasal quadrants of horizontal scans and also found higher diagnostic performance. Narayanaswamy $\mathrm{et}_{\mathrm{al}} \mathrm{l}^{7}$ reported an AUC of 0.90 for the AOD750 (angle opening distance at $750 \mu \mathrm{m}$ from the scleral spur), while Park et $a l^{8}$ showed an AUC of 0.99 for

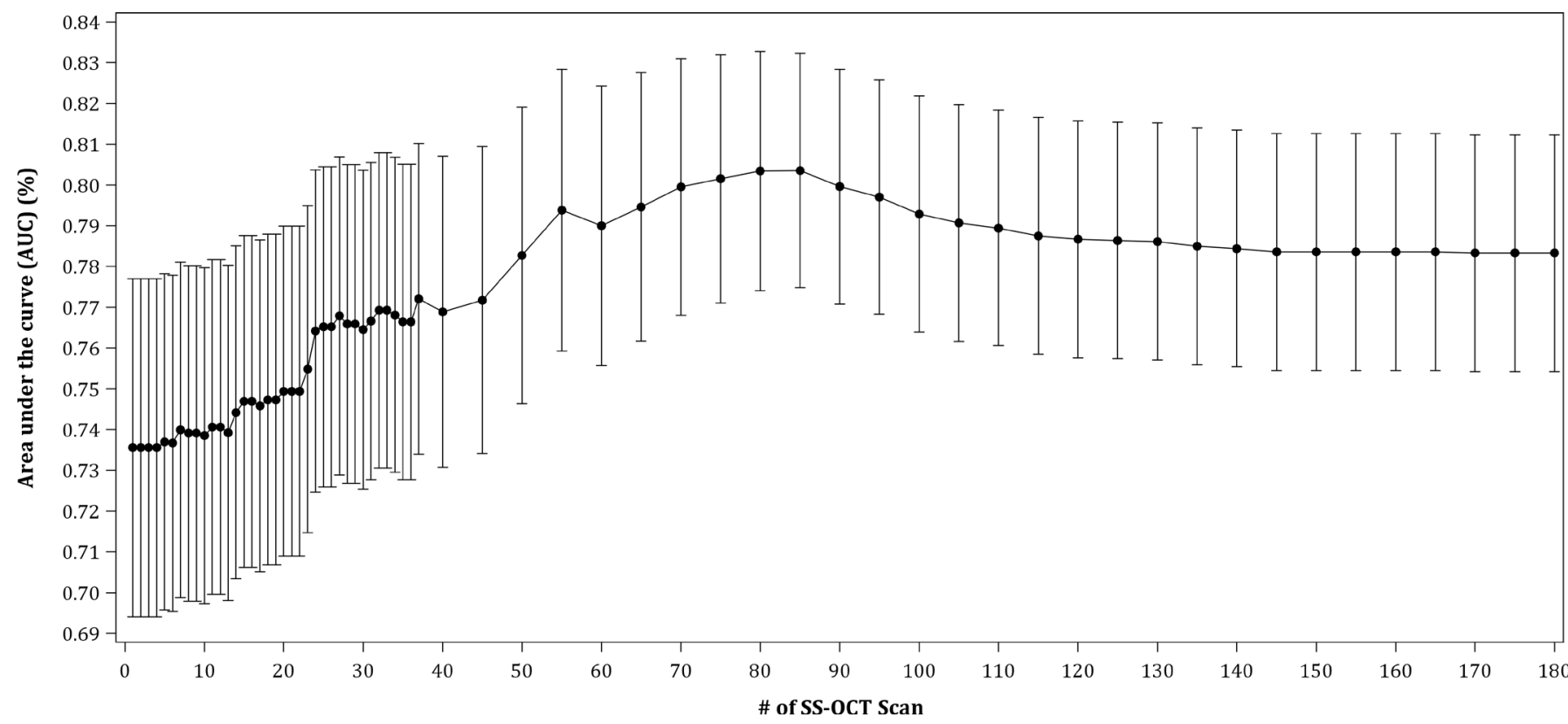

Figure 3 Cumulative effect of single SS-OCT. Cumulative diagnostic performance of the meridional SS-OCT scans consecutively added for the twoquadrant gonioscopic angle closure. AUC, area under the receiver operating characteristic curve; SS-OCT, swept-source optical coherence tomography. 
Table 1 Diagnostic performance of combinations of SS-OCT single scans for two-quadrant gonioscopic angle closure

\begin{tabular}{lll}
\hline Combinations of SS-OCT scans & AUC & $95 \% \mathrm{Cl}$ \\
\hline Horizontal (0-180)-vertical (90-270) & $0.8031^{*}$ & 0.7738 to 0.8325 \\
\hline Diagonal 45 (45-225)-diagonal 135 (135-315) & $0.8001^{*}$ & 0.7696 to 0.8305 \\
\hline Horizontal-vertical-diagonal 45-diagonal 135 & $0.7922^{*}$ & 0.7634 to 0.8211 \\
\hline
\end{tabular}

${ }^{*} P<0.001, \chi^{2}$ compared with scan $80^{\circ}-260^{\circ}$ (AUC 0.82).

AUC, area under the receiver operating characteristic curve; SS-OCT, swept-source optical coherence tomography.

AOD500 (angle opening distance at $500 \mu \mathrm{m}$ from the scleral spur).

Interestingly, in a previous study with Visante time domain AS-OCT, we showed that including the inferior angle only (at $270^{\circ}$ ) had the best diagnostic performance (AUC 0.76) for angle closure detection. ${ }^{9}$ From an optimal quantitative assessment perspective, Blieden et $a l^{10}$ reported that 16 meridional scans equally separated by $32^{\circ}$ are enough to calculate trabecular iris circumferential volume at $750 \mu \mathrm{m}$, and eight meridional scans to estimate iris volume, two useful parameters to assess anterior chamber angle configuration. Whether these quantitative parameters can be accurately measured in fewer scans remains to be studied. This and our analysis are non-conservative ways of evaluating gonioscopic angle closure with AS-OCT imaging in view of optimising the diagnostic process at expenses of the variability of the condition (ie, percentage of the circumference closed).

Our study shows that scans of the superior-inferior area are the most discriminatory and play a large part in the overall diagnostic performance of SS-OCT compared with the temporonasal scans. This can be explained by the fact that the superior quadrant has the highest rates of angle closure, followed by the inferior quadrant, both by gonioscopy and AS-OCT imaging. ${ }^{11}$ In the current study population, the superior quadrant was also found to have the highest rate of angle closure. ${ }^{2}$ When comparing horizontal with vertical, SS-OCT slightly overcalls more the vertical than the horizontal meridians (vertical overcalls 35.48\% (659/1857) vs horizontal overcalls $28.80 \%$ (535/1857)). Thus, the lower AUC for horizontal scans can be explained by the fact the horizontal scans and horizontal quadrants on gonioscopy had lower rates of angle closure. Similar observations with regard to the rates of angle closure in the superior quadrant have been made by other authors using gonioscopy ${ }^{12} 13$ and ultrasound biomicroscopy. ${ }^{14}$ The combination of the vertical-horizontal SS-OCT scans $\left(0^{\circ}-180^{\circ}\right.$ and $\left.90^{\circ}-270^{\circ}\right)$ performed better than those the reported with Visante (AUC $0.80,95 \%$ CI 0.77 to 0.83 vs AUC $0.72,{ }^{9} 95 \%$ CI 0.704 to 0.745 ), but this scan combination had still inferior performance than the single $80^{\circ}-260^{\circ}$ SS-OCT scan in the current study.

Our study has some limitations, such as the potential error in manual grading of images, reliance on a single person's gonioscopy and use of only a Sussman lens as opposed to a Goldmann lens (which may have decreased the rate of identified angle closure due to compression on the cornea opening the angle). As this was a community-based study, there exists the potential for some inherent sampling bias as we included patients in a primary care setting in Singapore. We do not know if the scanning optimisation proposed here is generalisable to an eye clinic setting where the prevalence of angle closure would be higher and more severe disease may be present. Moreover, SS-OCT remains suboptimal to perfectly discriminate angle closure based on gonioscopy. Lastly, the test was only done on the right eyes, and we can only assume that the mirror image scan would be equally as good for the left eye.

In conclusion, our study suggests that the theoretical advantages of SS-OCT, such as $360^{\circ}$ evaluation of the anterior chamber angle, do not translate to better clinical utility for detection of gonioscopic angle closure. The superoinferior scans and particularly the single scan at $80^{\circ}-260^{\circ}$ had the highest diagnostic performance to classify angle closure.

Contributors Conception and design of the work: NP, MB, SP, DF, CYC and TA. Acquisition, analysis or interpretation of data for the work: NP, MB, TAT, RS, MT, JHQ, JCA and TA. Drafting of the work: NP. Revising the work: NP, MB, SP, DF, CYC, TA, TAT $\mathrm{RS}, \mathrm{MT}$, JHQ and JCA.

Funding This work was supported by National Medical Research Council and Biomedical Research Council, Singapore (grant number 10/1/35/19/674).

Competing interests None declared.

Patient consent for publication Not required.

Ethics approval The study had the approval of the SingHealth ethics review board and was performed in accordance with the tenets of the Declaration of Helsinki.

Provenance and peer review Not commissioned; externally peer reviewed. Data availability statement Data are available upon reasonable request.

ORCID iD

Tin Aung http://orcid.org/0000-0002-7916-0589

\section{REFERENCES}

1 Sakata LM, Lavanya R, Friedman DS, et al. Assessment of the scleral Spur in anterior segment optical coherence tomography images. Arch Ophthalmol 2008;126:181-5.

2 Porporato N, Baskaran M, Tun TA, et al. Assessment of circumferential angle closure with Swept-Source optical coherence tomography: a community based study. Am J Ophthalmol 2019;199:133-9.

3 Baskaran M, Ho S-W, Tun TA, et al. Assessment of circumferential angle-closure by the iris-trabecular contact index with swept-source optical coherence tomography. Ophthalmology 2013;120:2226-31.

4 Ho S-W, Baskaran M, Zheng C, et al. Swept source optical coherence tomography measurement of the iris-trabecular contact (ITC) index: a new parameter for angle closure. Graefes Arch Clin Exp Ophthalmol 2013;251:1205-11.

5 Lavanya R, Foster PJ, Sakata LM, et al. Screening for narrow angles in the Singapore population: evaluation of new noncontact screening methods. Ophthalmology 2008;115:1720-7.

6 Tun TA, Baskaran M, Tan SS, et al. Evaluation of the anterior segment Angle-to-Angle scan of Cirrus high-definition optical coherence tomography and comparison with gonioscopy and with the Visante OCT. Invest Ophthalmol Vis Sci 2017:58:59-64.

7 Narayanaswamy A, Sakata LM, He M-G, et al. Diagnostic performance of anterior chamber angle measurements for detecting eyes with narrow angles: an anterior segment OCT study. Arch Ophthalmol 2010;128:1321-7.

8 Park SB, Sung KR, Kang SY, et al. Assessment of narrow angles by gonioscopy, van Herick method and anterior segment optical coherence tomography. Jpn J Ophthalmol 2011:55:343-50.

9 Khor W-B, Sakata LM, Friedman DS, et al. Evaluation of scanning protocols for imaging the anterior chamber angle with anterior segment-optical coherence tomography. J Glaucoma 2010;19:365-8.

10 Blieden LS, Chuang AZ, Baker LA, et al. Optimal number of angle images for calculating anterior angle volume and iris volume measurements. Invest Ophthalmol Vis Sci 2015;56:2842-7

11 Sakata LM, Lavanya R, Friedman DS, et al. Comparison of gonioscopy and anterior segment ocular coherence tomography in detecting angle closure in different quadrants of the anterior chamber angle. Ophthalmology 2008:115:769-74.

12 Scheie HG. Width and pigmentation of the angle of the anterior chamber; a system of grading by gonioscopy. AMA Arch Ophthalmol 1957:58:510-2.

13 He M, Foster PJ, Ge J, et al. Gonioscopy in adult Chinese: the Liwan eye study. Invest Ophthalmol Vis Sci 2006;47:4772-9.

14 Kunimatsu S, Tomidokoro A, Mishima K, et al. Prevalence of appositional angle closure determined by ultrasonic biomicroscopy in eyes with shallow anterior chambers. Ophthalmology 2005;112:407-12. 